\title{
Educación y Trabajo en Ciencias de la Educación: Aportes desde el Estudio de las Trayectorias Profesionales de sus Graduados
}

\author{
María E. Vicente \\ Facultad de Humanidades y Ciencias de la Educación, Universidad Nacional de La Plata (UNLP), \\ Consejo Nacional de Investigaciones Científicas y Técnicas (CONICET). Calle 9 No. 586, \\ CP 1900, La Plata-Argentina (e-mail: mevicente@fahce.unlp.edu.ar)
}

Recibido May. 28, 2012; Aceptado Jul. 05, 2012; Versión final recibida Jul. 12, 2012

\begin{abstract}
Resumen
Se ha estudiado la relación entre educación y trabajo en ciencias de la educación a partir del análisis de las trayectorias profesionales de sus graduados. Desde un método cualitativo de investigación biográfico narrativo, se realizaron entrevistas a graduados en Ciencias de la Educación de la UNLP en Argentina. Los resultados obtenidos a partir del análisis de contenido muestran que los graduados a lo largo de sus trayectorias reflexionan sobre sus historias escolares y familiares, diseñan su propio itinerario profesional, construyen nuevos espacios de acción laboral y enfrentan resistencias del entorno social. Como conclusión, las prácticas y estrategias desarrolladas a lo largo de la trayectoria profesional tienen la capacidad de reconstruir el campo profesional de las Ciencias de la Educación.
\end{abstract}

Palabras clave: educación, trabajo, trayectorias profesionales, ciencias de la educación

\section{Education and Work in Science Education: Contributions from the Study of the Professional Trajectories of its Graduates.}

\begin{abstract}
The relationship between education and work in Science Education from the analysis of the professional trajectories of its graduates has been studied. A qualitative method of narrative biographical research and interviewing graduates of Science Education from the National University of La Plata in Argentina. The results obtained from content analysis show that graduates reflect on school and family histories, design their own career, build new areas of pedagogical action, and face resistances. In conclusion, practices and strategies developed along the professional trajectory have the ability to reconstruct the professional field of Science Education.
\end{abstract}

Keywords: education, work, professional trajectories, science education 


\section{INTRODUCCIÓN}

Hacia principios del siglo XX, la complejidad creciente de los problemas sociales exigió un cúmulo de saber más variado y complejo para su solución, produciéndose una transformación radical en las formas típicas de reproducción y producción de los conocimientos, y con ello, en las formas de transmisión. De esta manera, el aprendizaje del saber formalizado ya no transcurría de forma espontánea sino que era materia de cálculo y planeamiento. La enseñanza se transformó así en una práctica específica, poniendo en funcionamiento medios adecuados a la finalidad de formación.

Enseñar se convirtió en tarea de especialistas y profesionales, naciendo entonces la profesión de maestro y junto a ella un saber muy específico: la pedagogía (el saber enseñar), objeto de estudio de las Ciencias de la Educación. Al respecto, sostiene Novoa: "Se está frente a saberes que se aplican en la formación profesional de los docentes y que servirían para justificar la posición de expertos y el monopolio de la intervención en el campo educativo" (1997: 250). En particular, la constitución de los profesionales en Ciencias de la Educación se relaciona con la necesidad de atender la enseñanza para los sistemas escolares públicos de ámbito nacional, manteniendo a dichos profesionales ligados al ejercicio dentro del marco escolar, particularmente la docencia (Armengol, 2005).

En el caso particular de Argentina, el campo profesional de las Ciencias de la Educación se funda en 1914 cuando se crea la Carrera y Facultad de Ciencias de la Educación en la Universidad Nacional de La Plata; referente obligado al momento de hablar de la formación docente a principios del siglo XX (Southwell, 2003).

En 1960, dicha universidad fue sede de la Primer Reunión de Departamentos e Institutos Universitarios Nacionales de Ciencias de la Educación en la que, entre otros temas, refirieron al campo profesional para el ámbito nacional, reconfirmando su posición en el campo formal de la educación:

"Que deben ser objetivos profesionales básicos los siguientes:

a. La preparación de profesionales para conducir y asesorar los organismos rectores de la educación en los distintos niveles de la enseñanza.

b. La preparación de profesionales para la investigación de base y de campo en los dominios de la educación.

c. La preparación de docentes para la enseñanza media y superior" (RACE, 1961).

Hacia 1980 en Argentina, la reconfiguración del campo se hacía presente en los documentos curriculares, tal como lo muestra el plan de estudios de Ciencias de la Educación de la Universidad Nacional de La Plata:

"Las asignaturas incluidas en el plan tienden a la habilitación de graduados en Educación para desempeñar responsabilidades inherentes a la planificación, conducción y asesoramiento en los niveles del macrosistema educativo, en los sistemas formales y no formales de educación, en el ámbito cultural y social y en los microsistemas y el trabajo psicopedagógico, así como en la administración de establecimientos e instituciones educacionales de diversa índole" (Plan de Estudios 1986, modificando plan 1978, FaHCE, UNLP).

En la década del 90, los espacios de acción laboral del pedagogo se diversifican y se ejercen en distintos escenarios, no sólo dentro de la educación formal sino también dentro de escenarios "no formales" e informales, tales como empresas, educación de adultos, consultoría, entre otros.

Ya a principios del siglo XXI, el campo profesional de las Ciencias de la Educación se constituye de diversos espacios sociales, además del escolar. Romaní y Zaragoza (2008) los identifican como educación institucional y educación en otros contextos. Como educación institucional reconoce los espacios de docencia; dirección, coordinación y asesoramiento; asesoramiento en programas educativos; investigación educativa; en administración educativa. Respecto de los 
contextos no formales refieren a la empresa, en editoriales, TIC y medios de comunicación, salud, medio ambiente (educación ambiental), dirección y gestión pública de servicios; en servicios sociales (atención y prevención de problemáticas sociales), sociocultural y sociocomunitario.

Así, la enseñanza y formación docente en tanto ámbito de actuación original ha quedado reducido, al mismo tiempo que intenta reconstituirse a partir de espacios emergentes. El campo socioprofesional de las Ciencias de la Educación se ha ido construyendo históricamente como un desprendimiento por fuera del sistema educativo, de la propia profesión docente (Testa y Spinosa, 2009). En el marco de la diversificación de espacios de acción pedagógica, del origen de nuevos espacios laborales y de la desaparición de otros tradicionales, vale preguntarnos por el propio graduado, ¿cuáles son las prácticas y estrategias socio-profesionales que desarrollan con el devenir del campo laboral educativo? En otras palabras, interrogamos por la relación entre educación y trabajo.

De la empleabilidad a las estrategias socio-profesionales

Varias son las perspectivas de abordaje al estudio de la relación entre educación y trabajo para el caso de los profesionales en Ciencias de la Educación. Los estudios realizados en Austria por Altrichter (1982); en España por Ventura y Martínez (2005); en Argentina por Carrió de Scaccia (2003), y Godino y Bongiovanni (2000), entre otros, responden a la idea de "empleabilidad" de los graduados, focalizando en las demandas de los empleadores y el grado de pertinencia de la formación universitaria en relación con las necesidades del mercado laboral. Por ello, esta primera perspectiva está orientada por el objetivo de diagnosticar las tendencias de ocupación, tiempo que se insume en la inserción laboral, la satisfacción respecto de la formación recibida y la aplicación de los conocimientos en el empleo (Hernández, Tavera y Jiménez, 2012). En este sentido, el supuesto que subyace a este abordaje se erige sobre la base de entender que el perfil del graduado debe necesariamente constituirse por y para las necesidades laborales que demanda el mercado de trabajo.

No obstante, los estudios realizados en México por Navarrete Cazales (2008); en Argentina por Testa y Spinosa (2009) e Ilvento (2009), entre otros, responden a una segunda perspectiva de tipo subjetivista. Sostienen que la función social de la educación no se reduce a ser la proveedora puntual de los recursos humanos que requieren los mercados de trabajo. Al mismo tiempo, los agentes sociales no son partículas mecánicamente tironeadas de aquí para allá por fuerzas externas, sino que tienen capacidad de elección y acción. De esta manera, esta segunda línea de análisis sostiene que, para analizar el trabajo profesional de los graduados, necesariamente el foco de interés debe versar sobre el actor mismo. Esto es, el campo profesional de la Educación debe ser reconstruido y analizado a partir de las expectativas y representaciones que los graduados tengan sobre su campo laboral.

Entonces, hasta aquí reconocemos una Perspectiva de tipo Estructuralista que aborda al campo profesional desde y en función del contexto; y una Perspectiva Subjetivista, emplazada en un enfoque de tipo psicológico - congnitivista, que reconocen a las representaciones, ideas y expectativas del individuo como moldeadores de los espacios profesionales.

A partir ( $\mathrm{y}$ a diferencia) de estos encuadres conceptuales, una tercera perspectiva retoma los aportes de las dos anteriores y desde el prisma conceptual de trayectorias profesionales, aborda la relación entre actor y contexto. Las trayectorias se constituyen a partir de la construcción de coherencia y sentido que los actores hacen de sus prácticas, estrategias, en torno de lógicas de acción que corresponden a elementos del sistema y se han impuesto a los actores. Pero también, los agentes o sistemas de agentes que forman parte del campo pueden describirse como fuerzas que se oponen y se agregan, confiriéndole su estructura específica en diferentes momentos a lo largo del tiempo (Bourdieu, 1997; Dubar, 2002).

De esta manera, el análisis de las estrategias y prácticas que los actores realizan en cada uno de los espacios laborales y a través de ellos, nos permitirá conocer no sólo de qué manera el campo 
profesional configura y determina sus trayectorias, sino también las acciones desplegadas por los profesionales que inciden en la re-configuración del campo mismo.

\section{METODOLOGÍA}

El método elegido es cualitativo de tipo biográfico narrativo. Reconociendo a la historia oral como fuente de información donde los profesores cuentan su historia profesional en relación con los contextos sociales, familiares o escolares (Bolívar, Domingo y Fernández, 2001), la obtención de datos se realizó a través de una entrevista en profundidad cuya pregunta disparadora del relato era: ¿cómo organizarías el relato de tu historia profesional?

Las entrevistas fueron realizadas a doce (12) graduados de la carrera de Ciencias de la Educación de la Universidad Nacional de La Plata (UNLP), egresados de las décadas de 1980, 1990 y 2000. Para la selección de los graduados se utilizó: I) el criterio de accesibilidad y diversidad de década de egresos. II) Respecto del criterio para determinar la cantidad de entrevistados, se relacionó con el nivel de saturación en las respuestas: cuando en las respuestas se encontraban ciertas relaciones o se reconocían determinadas categorías analíticas se cerraba el número de entrevistados. III) Finalmente, se seleccionó la carrera de Ciencias de la Educación de la UNLP por un criterio fundacional, es en dicha institución donde se abre la carrera por primera vez en Argentina, en 1914.

A través del instrumento se recogieron datos respecto de epifanías o incidentes críticos (Bolivar, Domingo y Fernández, 2001) que manifiestan aquellos eventos en la vida individual, seleccionados en función de que marcaron particulares rumbos, acontecimientos y cambios. Así, la idea de transformación forma parte central de la forma biográfica y se relaciona con el enfoque conceptual sostenido en el presente estudio, donde se reconoce la capacidad de los actores de modificar la realidad.

El análisis de las entrevistas es de tipo categórico del contenido (Lieblich, Tuval-Mashiach y Zilber, 1998). Categórico porque los fragmentos del relato fueron clasificados en diferentes categorías o grupos de análisis, y de contenido porque se focalizó en lo que sucedió, cómo, por qué, personas que intervinieron, etc. De esta manera, el análisis de los datos recolectados se analizaron en función de: a) de qué manera presentan su propia historia profesional; b) qué tipo de propuestas de mejora o cambios desarrollaron en los espacios laborales y por qué, c) cómo fueron recibidas dichas acciones, cuál fue el impacto en el grupo de pares, d) cómo y por qué deciden abrir nuevos espacios de acción pedagógica inexplorados previamente, y quiénes intervinieron; e) qué dinámicas contextuales e institucionales marcaron o delimitaron las prácticas profesionales.

\section{RESULTADOS}

\section{Los graduados y sus trayectorias}

Las biografías, anuncia Anijovich (2009), tratan de narrativas realizadas por la propia persona siguiendo un sistema de elaboración que hay que tratar de reconocer para otorgarle significación. Narrar la propia historia profesional no tiene que ver únicamente con fechas, sino con aquellas imágenes que reflejan de alguna manera la forma en que los entrevistados se piensan y piensan su trayectoria. Las lecturas que se hagan de ellas, sostendrán Nicastro y Greco (2009) invitan a entender las historias, no como la historia de alguien con inicio y final, sino como configuraciones que se anudan en diferentes puntos. En nuestro caso, podemos reconocer que índices, contenidos, temas, momentos o etapas son estructuras que le dan sustento a la organización de las trayectorias profesionales, por ejemplo:

"Describiría mi trayectoria en dos grandes momentos, incluyendo determinados hitos. Primero hay
un momento confuso, que es un universo de cosas, que forman parte de mis intereses pero que
yo ni siquiera le puedo poner nombre, al principio esto no se llamaba ni educación no formal, ni
educación en los museos, ni pedagogía social, ni todo eso que aparece después. Es una primera
etapa en la que voy haciendo cosas que me gustan porque ya leí en las incumbencias 
profesionales que, eran tan amplias, y pensé que algo de lo que me gustaba se relacionaría con la educación. Un primer momento de universo de intereses que no tiene un nombre. También hubo hitos, por ejemplo, darme cuenta que había gente con mi título que hacía lo que a mí me interesaba o ver que existía en otras carreras o en otras universidades que existía el espacio de la educación no formal. Esto me hace ordenar un poco más la cosa. Es el momento posterior a mi recibida. Me recibo y se me empiezan a juntar un poco más las ideas. Al principio tenía que convencer a la gente que lo que yo hacía tenía que ver con la educación, que también podía ser abordado desde una perspectiva pedagógica. $Y$ hubo un segundo momento en que yo sentí que no tenía que convencer a nadie de eso y que habían empezado a entender que eso que yo hacía también tenía que ver con la educación, y me empiezan a aparecer trabajos específicos" (4.B.)

Narrar la propia trayectoria se relaciona con aquellos referentes que el graduado va reconociendo para su práctica profesional, a lo largo de su trayectoria. En un primer momento se relacionan con las incumbencias profesionales, con lo normativo, con aquello que está escrito y que indica qué es lo que puede hacer el graduado en Ciencias de la Educación. Así, las incumbencias profesionales aparecen en un primer momento como criterio que definiría la práctica y que coincide con el ingreso a la carrera.

Luego reconocemos un segundo momento, coincidente con el egreso, y tiene que ver con reconocerse en escena no ya en los documentos, en lo normativo, en lo que debería ser; sino más bien en la práctica, en lo que "está siendo". Y para ello buscará en el colectivo profesional, en los mismos colegas: "había gente con mi título que hacía lo que a mí me interesaba o ver que existía en otras carreras o en otras universidades el espacio de la educación no formal".

Así, en un primer y segundo momento de la historia profesional del graduado, el referente de su práctica se va desligando de la norma, del deber ser, y se torna hacia las prácticas y sujetos concretos. Como veremos más adelante, este giro es clave para la construcción de nuevos espacios de acción pedagógica o la consolidación de otros que están emergiendo, o bien para el desarrollo de innovadoras propuestas pedagógicas. Y ello se relaciona con un tercer momento, que implica abandonar la idea de sujeto como ejecutor de normas, para reconocerse constructor y diseñador de su propio espacio de acción.

En relación con la descripción de los momentos en las historias de vida profesionales, Michael Huberman (1990) habla de "fases de la profesión docente" que van desde la entrada a la carrera hasta la fase final llamada "de ruptura", pasando por etapas de estabilización, replanteamiento y distancia. Por su parte, Sikes (en García, 1999) refiere a "ciclos de vida" en la carrera docente, que comprende las etapas de exploración, transición, estabilización hasta la jubilación. Finalmente, Lombardi y Vollmer (2009) denominan "desarrollo profesional" al proceso a través del cual se logra que los saberes prácticos de los docentes se articulen con el saber experto acumulado, con las experiencias desarrolladas por otros colegas. En este marco, nos animamos a presentar nuestras historias de vida profesional como "biografías en referencia" que trata sobre aquellos referentes en los que el graduado se apoya o reconoce a lo largo de su vida profesional, y que van desde un importante apego a lo establecido, desde las normas, hacia la búsqueda de nuevas maneras de hacer y pensar la práctica.

\section{Biografías escolares: estructuras cuestionadas.}

Según los aportes de Delory - Momberger (2009), los individuos de las sociedades posmodernas se ven rehaciendo la planificación y gestión de su recorrido biográfico. En las formas de organización social donde la autonomía y la responsabilidad individuales se tornaron valores sociales y económicos, compete a los sujetos probar "biográficamente" su formación y empleo. De cierto modo, el actor social pasó de la ejecución de acciones programadas y papeles establecidos a "comandar" un conjunto de lógicas posibles y soluciones alternativas, a partir de los cuales debe explorar su propio itinerario, orientando y adaptando su "dirección":

"Fui a un colegio de monjas muy cerrado, estructurado. Y sentía la necesidad de liberación, no quería nada que me estructura, nada de estructuras cerradas. No quería que me dieran un 
programa y que tenía que dar eso. Yo quería tomar decisiones. En Capacitación laboral lo que más me gustaba era las entrevistas con los expertos en contenidos y luego yo tomaba decisiones respecto del diseño" (7.D.)

"Estudiar Ciencias de la Educación en una universidad pública se relacionaba con demostrarme a mí misma que yo podía hacerlo sola. En mi casa yo tenía el mundo tan resuelto, que yo necesitaba demostrarme a mí misma que yo podía elegir y tomar decisiones sola. (...) Mi universo era la escuela de monjas, de todas mujeres solamente, donde de algunas cosas en mi casa no se hablaba" (12.A.)

Como podemos notar en estas líneas, el "direccionar" el itinerario socioprofesional tiene que ver con cuestiones de orden familiar y con instituciones por las que han transitado a lo largo de sus vidas, en particular hacemos referencia a la escuela. Al respecto, Delorenzi (2008) se refiere a la práctica profesional del sujeto en tanto proceso de construcción que está atravesada por tradiciones de formación históricamente determinadas, y por experiencias y recuerdos escolares individuales, impregnados de éxitos y de fracasos, de mitos y de rituales, de formas de sentir y de percibir. Por su parte, los autores Palacios y Cárdenas (2008) referirán al rol de las instituciones en el contexto actual para pensar las biografías individuales. Sostienen que asistimos a un debilitamiento del contenido normativo de las instituciones y, consecuentemente, una expansión de las posibilidades de elección y decisión de los sujetos. La disolución de las formas de vida propias de la sociedad industrial hace que los sujetos ya no puedan cumplir con la tradicional "trayectoria de vida" y bajo las cuales se estructuraron las instituciones básicas de socialización (por ejemplo, la escuela, la familia). Ahora los sujetos deben asumir las responsabilidades y posibilidades, los riesgos e (in)seguridades relativos a la definición, planificación y organización de su propia forma de vida y de sus relaciones sociales.

En nuestros casos, la experiencia del tránsito por la escuela de la infancia y la necesidad de cambio se entrelazan al momento de decidir ingresar a la carrera de Ciencias de la Educación, carrera que consideran les puede dar las oportunidades vedadas en el pasado: "la posibilidad de decidir". "En mi casa tenía todo resuelto y la carrera me daba la posibilidad de resolver las cosas por mí misma". Así las posibilidades de acción futura no estaban determinadas desde los espacios socio educativos tradicionales como la escuela y la familia, sino en el propio sujeto, es el mismo graduado quien se hace cargo de la forma en que transitaría su carrera.

Ahora bien, hasta aquí reconocemos, a través de los aportes teóricos y de las voces de los entrevistados, el protagonismo del sujeto al momento de diseñar su itinerario profesional en el que las biografías escolares se presentan como el resultado de complejas internalizaciones durante la vida escolar que van generando un "fondo de saber" que regula las prácticas (Davini; 1995, 79). Proponemos considerar la idea de "fondo de saber que regula las prácticas" como un sustantivo posible de adjetivar, una frase incompleta, una palabra a ser convertida en acción: "fondo de saber que regula las prácticas que el sujeto es capaz de capitalizar y así redefinir la realidad"

\section{La posibilidad de construir la carrera: desafíos y cambios.}

Según el análisis hasta aquí realizado, la posibilidad de "hacerse cargo" de la práctica, diseñando itinerarios profesionales propios es posibilitado por, al menos, dos condiciones. Por un lado el sujeto, en tanto actor social, tiene la capacidad de modificar la realidad; al mismo tiempo que las instituciones y a través de ellas, las normas, en los últimos tiempos han perdido el poder de determinar la historia y destino de los sujetos. En este marco, es necesario rescatar la idea de cargo o rol:

"Los cargos están pero uno les da sentido, los roles se construyen. En la práctica comenzás a reflexionar. En mi caso, en los gabinetes psicopedagógicos encontrás tradiciones sobre las formas de abordaje e intervención. Muchos pensaban en términos de problemas de aprendizaje, y nosotros pensábamos que los problemas también se relacionan con la enseñanza misma. O sea, desde la enseñanza, poder reforzar esos problemas de aprendizaje. Hubo un cambio de paradigma que tiene que ver con ir instalando de a poco tu mirada, para construir la identidad del 
rol. Y acá no sólo atendíamos al niño en contraturno, sino que también fuimos a trabajar con el maestro al aula. En la práctica vas construyendo" (5.C.)

Según podemos reconocer en las palabras antecedentes, los cargos y roles cobran identidad en tanto son producto de los significados atribuidos por el profesional. Por ello, en lugar de hablar de cargo preferimos referirnos a aquel marco de acción y sentido desde donde el graduado piensa la realidad. Situación que es facilitada, según Tenti (1990), por las transformaciones sociales que han roto la relación de correspondencia entre profesión y problema a resolver. Condición que se constituye en límites para las acciones y recursos para las mismas, abren ciertas posibilidades y cierran otras, implican prohibiciones pero también habilitan para actuar. Las acciones que los actores desarrollan en el desplazamiento por el campo profesional, están condicionadas pero también tienen capacidad de opción, de elección y de reflexión sobre lo que hacen (Dubet, 1994). Así, la posibilidad de desarrollar cambios en los ámbitos laborales, es una dimensión que se presenta en todas las historias a las que pudimos acceder. Retomamos una de ellas:

"En mi vida profesional hubo un punto de inflexión. Fue en tercer año de la carrera, con la apertura de la democracia [1983], cuando curso sociología de la educación. A partir de allí comienzo a participar políticamente desde la Comisión de Estudiantes para la reforma del plan de estudios. Al mismo tiempo yo trabajaba como maestra de nivel primario en la escuela de la universidad, y allí había un grupo de tres docentes, conocidas como las "transgresoras", y me empiezo a acercar a ellas. A este grupo yo llevaba los apuntes de la facultad, y llevé cuestiones nuevas de la didáctica. A partir de allí hicimos un trabajo experimental en el área de matemática. Rompimos lógicas, y comenzamos a trabajar con teoría de conjuntos. A partir de allí, comenzamos a planificar el área de ciencias sociales por problemas: trabajo, hambre, población. Categorías aglutinantes que permitía dar otros contenidos. Esto fue para todas las divisiones de sexto grado. Estos chicos cuando rindieron el examen para ingresar a la secundaria de la universidad, claramente presentaban los mejores rendimientos. En mi caso, hacer esto se relacionaba con lo que estudiaba en la carrera. Yo me estaba formando en el área sociológica. Tiempo después la única que quedó del grupo fui yo, y esa experiencia me convirtió en una voz autorizada. Me pedían opinión o me encargaban trabajos especiales, por ejemplo, coordinar la comisión de convivencia o la de trabajo curricular" (2.G.)

Hasta aquí el análisis de las historias nos ha permitido pensar al sujeto como actor social, creador de su propio recorrido profesional, siendo capaz de ampliar el horizonte de posibilidades en la práctica.

\section{Acciones Pedagógicas y Resistencias}

En este apartado intentaremos aproximarnos a las consecuencias que las acciones pedagógicas de los graduados en Ciencias de la Educación tienen en los espacios de trabajo, reconociendo que existen diferentes actores implicados en el campo educativo, que actúan destacando y opacando asuntos según sus núcleos de intereses y su reserva de conocimientos, y ello se traduce en el campo profesional:

"A fines del 90, cuando cambia la gestión del Ministerio de Justicia y Seguridad, me convocan para trabajar en el instituto superior de formación policial, y me ofrecen el cargo de coordinadora académica. Cuando entro a trabajar allí comienzo a iniciar algunos cambios, por ejemplo, trabajar con los docentes que no iban a dar clases, comenzar a realizar talleres de capacitación con ellos, y prensando en propuestas con contenido. Hasta ese momento, las propuestas estaban vacías de contenido. Como respuesta a eso, tuve una clara resistencia o intento de que yo desista. Un día, en toda la institución repartieron un folleto con un dibujo desagradable y la imagen de mi cara. Luego de eso, pensé que si yo me iba de esa institución era porque yo así lo decidía, no porque algunos me querían afuera. Entonces decidí quedarme y continué con mi trabajo y las propuestas de talleres que yo había llevado a ese espacio" (10.D.).

"Cuando comenzamos con esta propuesta de enseñar matemáticas en el marco del aprendizaje significativo, había mucha desconfianza, principalmente en el trabajo colaborativo. Los chicos a cargo hacían trabajo autogestionado, grupal e individual, así aprendían a pensar con otros. 
Nosotras pretendíamos que sea un aprendizaje significativo, no una cuestión de repetir o memorizar. Y el resto de las docentes, que nos denominaban "las de sexto", nos criticaban aduciendo que los chicos así no aprendían las cuentas de matemática y que el trabajo en grupo no se podía evaluar. Asimismo, cuando desarrollamos estas nuevas estrategias didácticas para el área de matemática, fue en el marco de una gestión que nos permitió hacer eso bajo la excusa de que era una escuela de la universidad y se podría experimentar algunos cambios. Pero esto no duró mucho, cuando cambia la gestión, se proponen repartirnos en diferentes años, ya no trabajamos más todas juntas en sexto, sino que nos repartieron de cuarto a séptimo grado. Claramente pretendieron romper con el grupo, y así fue, esa división nos debilitó porque ya no estábamos en grupo pensando juntas, rompieron con el espíritu corporativo, nos diluyeron. Realmente se nos presentaron muchas resistencias y críticas" (3.F.).

A lo largo de estas líneas podemos reconocer el interjuego entre lo macro institucional y lo micro a nivel de la práctica individual de cada sujeto. Tal como nos orienta el estudio de Hernández, Tavera y Jiménez (2012) para el caso de la práctica profesional de los profesores principiantes en el ámbito de la enseñanza universitaria, estamos pensando en la gestión institucional en tanto factor que incide en las acciones de quienes trabajan allí. En nuestro caso, ambas entrevistadas traen a sus historias los cambios de gestión institucional, necesarios para explicar que ello contribuyó a lograr la implementación de nuevas estrategias didácticas para la enseñanza de una disciplina o, al contrario, que se constituyó en obstáculo para llevar adelante nuevas propuestas de capacitación. Esto nos ayuda a comprender que la gestión institucional puede actuar como facilitador u obstaculizador de los cambios emprendidos por los graduados en las instituciones. Sobre cuestiones de orden institucional y político ahondaremos en el próximo apartado.

\section{Condiciones externas: política y formas de contratación.}

Las estructuras objetivas externas que condicionan las trayectorias aluden a la estructura de las organizaciones, la posición que los agentes ocupan en ellas, la posición que ocupan en los diferentes campos sociales y en el espacio social global, las normas sociales, las políticas que se implementan, etc. (Bourdieu, 1997). En nuestro caso, las condiciones externas que se hacen presentes en las narraciones de los entrevistados corresponden particularmente a cambios de gobierno y cambio de equipos de gestión en las instituciones gubernamentales:

"En mi historia, hubo un cambio importante por cuestiones contextuales, en particular, cuestiones que tienen que ver con cambios en el gobierno y en los equipos de gestión de las instituciones gubernamentales. En este caso, en 2002 hubo un cambio de gestión en la institución de formación de policías, dependiente del Ministerio de Justicia y Seguridad. En esa institución, con el cargo de coordinadora académica, yo venía desarrollando diversas actividades con formato de diseño de capacitaciones y talleres de capacitación docente. Coordinaba acciones extracurriculares. Venían expertos a capacitarlos a los docentes en diferentes temas. Actividades muy interesantes. Cuando cambia la gestión, percibo que no tenía lugar para continuar mi trabajo, me identificaban con la "pro intervención", entonces pido el traslado. Y allí me trasladan a una Comisaría de City Bell, y allí continué con mi trabajo, con el mismo enfoque que yo defendía, y realmente pudimos lograr proyectos muy buenos en términos sociales, por ejemplo, el desarrollo de una ONG sobre violencia familiar" (2.C.).

"Los programas de educación en museos en los que trabajo, en términos jurídicos y administrativos, no están estructurados. En este universo no hay mecanismos formales para entrar. No hay listados para inscribirse, no hay concursos. Cosa que se traduce en los cargos, como en la universidad o en el instituto, que sos profesora o ayudante diplomado. Pero en Mundo Nuevo, por ejemplo, tengo un cargo de secretaria administrativa y un cargo de maestra. Esto es inestable, y siento una sensación de incomodidad. Yo tengo un arreglo con las horas que trabajo y las declaradas y sentís que estás mintiendo. Si declaro todo, estoy incompatible. En Mundo Nuevo no estoy en la gestión, soy la coordinadora del programa, pero mi cargo dice que soy secretaria. (...) en estos programas estás muy sometido a los cambios políticos. El intendente es el que nos da las oficinas, los lugares para trabajar. O si cambia el ministro de educación de la provincia, temblás" (4.G.). 
En el caso de la primera entrevistada, los condicionamientos externos tienen que ver con un cambio de gestión que condicionó su permanencia en la institución. Pero el condicionamiento no sólo es tal debido a las características y lineamientos de la nueva gestión, sino por su relación con el enfoque pedagógico con el que trabajaba la graduada: ella permanecía si desistía de utilizar un enfoque "pro-intervencionista". Claramente la práctica pedagógica estaba condicionada. No obstante, las imposiciones que inciden sobre cada elección que los sujetos realizan a lo largo de su trayectoria responden a complejas combinaciones que, fruto de determinadas estrategias, permiten conciliar, equilibrar y, a veces, anular dichas imposiciones (Bourdieu, 2004). Es así que la graduada eligió retirarse de la institución y luego de un pedido de traslado encontró un espacio donde continuar trabajando con el enfoque, el mismo que permitió desarrollar posteriormente una ONG contra la violencia familiar.

Otra cuestión a rescatar es la relacionada con la seguridad laboral. Al respecto, Castel (2009) señala que en los últimos tiempos vivimos una etapa de "desestabilización de los estables" respecto del trabajo. Un proceso de precarización laboral que atraviesa zonas antes estabilizadas por el empleo, de las que los profesionales en Ciencias de la Educación también forman parte. En este escenario, y atendiendo a lo expresado por las entrevistadas, se emplean para tareas de corta duración, de algunos meses, y así más fácilmente despedibles. Las oportunidades provisionales sin certidumbre de mañana hacen de estos profesionales "interinos permanentes", situación que acoge la instalación de la precariedad. Al respecto, la entrevistada describe su situación laboral con las palabras de "inestabilidad" e "incomodidad" haciendo alusión a su situación en un programa socio-educativo que forman parte de aquellos espacios emergentes en la última década. En otros términos, la Coordinación Académica de un programa educativo de enseñanza de las ciencias es homologable a un cargo administrativo, el de secretaria. Si bien no consideramos que exista una relación directamente proporcional entre nuevos espacios de acción pedagógica y la ausencia de regulación que impacte negativamente en la seguridad laboral, sí es posible reconocer que la construcción de nuevos espacios también debe atender a la cuestión de las condiciones de trabajo.

\section{DISCUSIÓN}

El estudio presentado se separa de aquellos que reconocen al sistema productivo como determinante de la formación de profesionales en educación, como también de aquellos que suponen al graduado despojado de contexto y condicionantes. Es a partir de la misma intersección entre la dinámica estructural del campo y las prácticas socio-profesionales de los graduados que el estudio interroga por las prácticas y estrategias de los graduados a lo largo de sus trayectorias.

Con referencia particular al enfoque de trayectorias profesionales, los avances aquí presentados se desligan de la concepción de trayectorias como sucesión de puestos de trabajo ocupados y tareas realizadas, tal como pueden ser descritos objetivamente desde un punto de vista externo. Más bien, reconoce que cada profesional las construye a partir de trazar y volver a trazar sentidos, espacios, posibilidades y respuestas en formas no lineales.

Respecto de la biografía escolar y familiar previa y su influencia en los distintos espacios de inserción socio profesionales del graduado, el estudio discute con la idea de continum o círculo reproductivo entre la biografía escolar del futuro graduado, su formación y la institución en la que se inserta como profesional. Más bien, teniendo más cuidado al hablar de círculo o prácticas reproductivas; y evitando caer en una subestimación del sujeto, podemos decir que las entrevistadas nos enseñan que las experiencias escolares y huellas familiares pueden ser puestas en discusión apuntando a redefinir el sentido de las prácticas.

Finalmente, respecto de la concepción de profesión, las acciones socio-profesionales narradas por las entrevistadas dan cuenta que es posible trabajar en orden a generar las necesidades sociales de los profesionales en Ciencias de la Educación, y no sólo esperar las demandas dependiendo únicamente del conjunto de empleadores que buscan controlar los términos, las condiciones y el contenido de los trabajos que desean les realicen. 


\section{CONCLUSIONES}

1) Las trayectorias profesionales se presentan como "biografías en referencia" que trata sobre aquellos referentes en los que el graduado se apoya o reconoce a lo largo de su vida profesional, y que van desde un importante apego a lo establecido, desde las normas, hacia la búsqueda de nuevas maneras de hacer y pensar la práctica.

2) Las biografías escolares y familiares de los graduados actúan como fondo de saber de las prácticas a partir del cual el actor es capaz de poner en discusión a lo largo de sus trayectorias.

3) La concepción de profesión más adecuada que le cabe a las Ciencias de la Educación es la de "profesionalización de las Ciencias de la Educación" para intentar dar cuenta de la idea de reinvención constante de los espacios socio-profesionales en toda acción pedagógica de cada graduado.

4) Los cambios emprendidos por los graduados en los distintos espacios laborales son acciones vinculadas con el contexto institucional. La gestión institucional actúa como facilitador u obstaculizador de dichos cambios.

5) Respecto de las regulaciones del trabajo y los espacios emergentes de acción pedagógica, comprendemos que la construcción de nuevos espacios debe, como tarea pendiente, atender a la negociación de mejoras en materia de seguridad laboral.

\section{REFERENCIAS}

Altrichter, H., Austrian graduates in Education and their jobs: an empirical analysis of the occupational distribution of university graduates. Higher Education, 11 (5), 499 - 510, (1982).

Anijovich, R., Transitar la formación pedagógica. Dispositivos y estrategias. Buenos Aires: Paidós, (2009).

Argüello, S., y otros 5 autores, Un estudio sobre trayectorias laborales de graduados en Ciencias de la Educación. V Encuentro Nacional y II Latinoamericano La Universidad como objeto de investigación. Tandil: UNICEN, (2009).

Armengol, C., Perfil y competencias de los pedagogos hoy. Documento curso Pedagogía, España, (2005).

Bolívar, a.; Domingo, J. y Fernández, M., La investigación biográfico-narrativa en educación. Enfoque y metodología. Madrid: La Muralla, (2001).

Bourdieu, P., Razones Prácticas. Barcelona: Anagrama, (1997).

Bourdieu, P., El baile de los solteros. Madrid: Anagrama, (2004).

Birgin, A., Reproducción y cambio en la formación docente en el Seminario "La investigación educativa en América Latina". Buenos Aires: FLACSO, (1991).

Carrió de Scaccia, M. del C., Correspondencia entre el perfil del egresado de la carrera de Ciencias de la Educación y la demanda laboral. Comunicación proyecto Instituto Ciencias de la Educación, Facultad de Filosofía y Letras, Universidad Nacional de Cuyo, (2003).

Castel, R., Las metamorfosis de la cuestión social. 1ra Ed. 5ta reimp. Buenos Aires: Ed. Paidós, (2009).

Davini, M. C., La formación docente en cuestión: política y pedagogía. Buenos Aires: Editorial Paidós, (1995). 
Delorenzi, O., Biografía escolar: ¿Determinante de las prácticas docentes o punto de partida para su construcción? Voces de la Educación Superior. 1 (2), 1 - 8, (2008).

Delory - Momberger, Ch., Biografía y educación. Figuras del individuo - proyecto. Buenos Aires: Editorial de la Facultad de Filosofía y Letras de la Universidad de Buenos Aires; Consejo Latinoamericano de Ciencias Sociales, (2009).

Diker, G. y Terigi, F., La formación de maestros y profesores: hoja de ruta. Buenos Aires: Paidós, (2003).

Dubar, C., L'articulation des temporalités dans la construction des identités personnelles: questions de recherche et problèmes d'interprétation. Temporalistes, 1 (44), (2002).

Dubet, F., Dimensions et figures de l'expérience étudiante dans l'université de masse. Revue française de sociologie, 35 (4), 511-532, (1994).

Fondon, I., Madero, M. J. y Sarmiento, A. Principales Problemas de los Profesores Principiantes en la Enseñanza Universitaria. Revista Formación Universitaria 3 (2), 21-28, (2010).

Freidson, E., Las teorías de las profesiones. Estado del arte. Perfiles Educativos, 23 (93), 28 - 43, (2001).

Furlan, A. y Pasillas, M. A., Investigación, teoría e intervención en el campo pedagógico. Perfiles Educativos, (61), 64 - 89, (1993).

Godino, C. y Bongiovanni, N. S., La paradoja del cambio y la exclusión. Un debate sobre la inserción laboral del pedagogo. Primer Congreso Latinoamericano de Educación Superior siglo XXI, (2003).

Hernández, C. A; Tavera, M. E. y Jiménez, M., Seguimiento de Egresados en Tres Programas de Maestría en una Escuela del Instituto Politécnico Nacional en México. Revista Formación Universitaria. 5 (2), 41-52, (2012).

Ilvento, M. C., Las representaciones sobre el campo profesional de las Ciencias de la Educación. La incidencia de las prácticas pre-profesionales. Tesis de doctorado. Argentina: Universidad Nacional de Salta; España: Universidad Rovira I Virgili, (2004).

Lieblich, A., Tuval-Mashiach, R. y Zilber, T., Narrative research. Reading, analysis, and interpretation. California: Sage Publications, (1998).

Nicastro, S. y Greco, M. B., Entre trayectorias. Escenas y pensamientos en espacios de formación. Buenos Aires: Homo Sapiens Ediciones, (2009).

Novoa, A., Profesionalización de docentes y Ciencias de la Educación. Educación y Pedagogía. 9 -10 (19-20), 251-288, (1997).

Palacios, M. y Cárdenas, A., Vínculo social e individualización: reflexiones en torno a las posibilidades de aprender. Revista de Sociología de la Universidad de Chile, (22), 65 - 85, (2008).

Plan de estudios Ciencias de la Educación 1986, Facultad de Humanidades y Ciencias de la Educación, Universidad Nacional de La Plata.

RACE Revista Archivos de Ciencias de la Educación, Departamento de Ciencias de la Educación, Universidad Nacional de La Plata. 3ra. época, 1 (2), (1961).

Romaní, J. y Zaragoza, M., La pedagogía profesional del siglo XXI. Educación XXI, (11), 133-154, (2008). 
Southwell, M., Psicología experimental y Ciencias de la Educación. La Plata: Editorial de la Universidad Nacional de La Plata, (2003).

Tenti, E. y Gómez, M., Universidad y Profesiones. Miño y Dávila. Buenos Aires, (1990).

Testa, J. y Spinosa, M., Las expectativas profesionales de los alumnos próximos al egreso de la Licenciatura en Ciencias de la Educación. Luján: Universidad Nacional de Luján, (2009).

Ventura, J. y Martínez, F., Estudio sobre la inserción laboral de los graduados en Pedagogía de la Universidad de Barcelona. XIV Jornadas de Economía de la Educación, Oviedo, (2005).

Villa, A. I.; Pedersoli, C. y Martin, M., Profesionalización y campo ocupacional de las Ciencias de la Educación. Archivos de Ciencias de la Educación. 3 (3) ,113 - 128, (2009). 